\title{
Concerning demographic limitations on the population growth rate of West Australian (Breeding Stock D) humpback whales
}

\author{
A. Brandão And D.S. ButTERWORTH \\ MARAM (Marine Resource Assessment and Management Group), Department of Mathematics and Applied Mathematics, \\ University of Cape Town, Rondebosch 7701, South Africa \\ Contact e-mail: anabela.brandao@uct.ac.za
}

\begin{abstract}
The upper bound of 0.126 on the maximum demographically possible annual growth rate for humpback whales that has standardly been imposed on recent applications of age-aggregated assessment models for this species in the IWC Scientific Committee, is based on an analysis that assumes steady age structure. It is conceivable that transient age-structure effects could admit greater population growth rates for short periods than suggested by such a bound. This possibility is addressed by developing an age-structured population model in which possible density dependent changes in pregnancy rate, age at first parturition and natural mortality are modelled explicitly, and allowance is made for the possibility of natural mortality increasing at older ages. The model is applied to the case of the west Australian humpback whale population (Breeding Stock D), for which breeding ground surveys over the 1982-1994 period provide a point estimate of 0.10 for the annual population growth rate. Results based upon the breeding population survey estimate of abundance of 10,032 in 1999 suggest that 0.12 is the maximum demographically feasible annual rate of increase for this stock over 1982-1994 if it is a closed population. This result is based on essentially the same parameter choices as led to the earlier $r=0.126$ bound, i.e. that in the limit of low population size the age at first parturition approaches five years from above, the annual pregnancy rate 0.5 from below, and the annual natural mortality rate 0.01 from above. Transient effects do not appear able to reconcile the observed rate of increase with less extreme values of demographic parameters than led to the previously imposed upper bound of 0.126 on the maximum possible annual growth rate. Although use of extreme values reported for demographic parameters for Northern Hemisphere humpback whale populations, rather than those considered here, would reduce this suggested maximum rate of 0.12 , the conclusion that transient effects have a very limited impact on observed population growth rates would be unlikely to change.
\end{abstract}

KEYWORDS: HUMPBACK WHALE; SOUTHERN HEMISPHERE; MODELLING; GROWTH; PREGNANCY RATE; PARTURITION

\section{INTRODUCTION}

Considerable debate has arisen over the extent to which the population growth rates suggested by time series of survey estimates of abundance for various South Hemisphere humpback whale populations are consistent with the bounds imposed by the species' demographics. More specifically, the upper bound on the maximum per capita annual growth rate, $r$, of 0.126 imposed on recent age aggregated model assessments of these populations in the IWC Scientific Committee has been questioned as perhaps too high (IWC, 2011).

The origin of this bound is calculations by Brandão et al. (1999), discussed further in Clapham et al. (2001), which relate population growth rates to biological parameter values. Essentially the higher the pregnancy rate (shorter the calving interval), the greater the annual survival rate and the lower the age at first parturition, then the higher the growth rate that the population can attain. The value of 0.126 selected as a bound corresponds to the following choices regarded as 'pushing the limits' for plausible values of biological parameters for humpback whales:

$$
\begin{array}{lll}
\rho & \text { (annual pregnancy rate) } & =0.5 ; \\
S & \text { (annual survival rate) } & =0.99 ; \\
a^{\text {mat }} & \text { (age at first parturition) } & =5 \mathrm{yrs} .
\end{array}
$$

The questioning of the $r=0.126$ bound in IWC (2011) arose primarily because at least some of these values were considered too extreme on the basis of estimates from various humpback whale populations. However, the calculations of Brandão et al. (1999) are based upon the assumption of a steady age structure. It is conceivable that over short periods of time (typically 1-2 decades), transient effects could lead to the attainment of higher population growth rates than indicated by the results of that paper.
Expressed another way, such effects might allow high point estimates of population growth rate to be reconciled with less extreme values of demographic parameters.

The purpose of this paper is to investigate this possibility for the specific case of the west Australian humpback whale population (Breeding Stock D). Results from five breeding ground surveys of this population over the period 1982-94 (IWC, 1996) suggest an annual increase rate (the slope parameter from a log-linear regression against year) of 0.10 (95\% CI: 0.03-0.18). This paper explicitly models possible density dependent changes in various biological parameters to determine to what extent this estimate is consistent with the feasible behaviour of a closed population of humpback whales.

\section{DATA}

The historic catch data used for these analyses are as agreed at a recent Southern Hemisphere humpback whale workshop (IWC, 2011). Two series are considered, the 'Core' and the 'Fringe' series (see Fig. 1), corresponding to different assumptions for the allocation of catches made in high latitude (south of $40^{\circ} \mathrm{S}$ ) feeding areas among breeding stocks (Table 1). The 'Core' series makes the conservative assumption that only catches between $80^{\circ}$ and $100^{\circ} \mathrm{E}$ come from Breeding Stock D, whereas the 'Fringe' series reflects the alternative extreme including all such catches between $50^{\circ}$ and $130^{\circ} \mathrm{E}$.

\section{METHODS}

The age-structured population model used for these computations is described in detail in the Appendix. For simplicity, sexes are not distinguished. 
Table 1

Fixed parameters in the model for the reference case model and variants $2 \mathrm{a}$ $4 \mathrm{~d}($ see text)

\begin{tabular}{lc}
\hline Parameter & Value \\
\hline$a_{r}$ & 4 yrs* \\
target year $(y)$ & 1999 \\
target population size $\left(N_{y}^{T}\right)$ & 10,032 \\
$\rho_{\max }$ & 0.5 \\
\hline
\end{tabular}

*The minimum legal length for humpback whale catches from 1950 to 1962 was $35 \mathrm{ft}$, which compares with lengths at maturity of $39.5 \mathrm{ft}$ for females and $38 \mathrm{ft}$ for males (Chittleborough, 1965). Chittleborough also reports the immature fraction of the catch varying from $18-56 \%$ for females and $3-51 \%$ for males. This suggests a typical age-at-first capture $(a)$ slightly less than the age-at-maturity. Given that this paper considers the age-at-firstparturition to range between 5 and 10 years, a choice of $a_{r}=4$ does not seem unreasonable.

The model is 'fit' to the data by adjusting the (initial) carrying capacity $K$ so that the population's trajectory hits a target total $(1+$, i.e. survey is taken to count all animals of age 1 and above, so that only calves are excluded) population of 10,032 in the year 1999, as estimated from surveys of the breeding grounds (Paxton et al., 2011).

The selectivity ogive for past catches is generally taken to be knife-edge at age $a_{r}=4$ (see Table 1). However, for some of the models age structure effects lead to an inability to hit the target value in 1999 because of extinction. In such cases, the age-at-first capture $\left(a_{r}\right)$ was reduced to two years to avoid this difficulty.

For a 'reference case' the maximum possible pregnancy rate for the population $\left(\rho_{\text {max }}\right)$ is taken to be 0.5 , corresponding to a minimum possible calving interval of two years. This applies in the limit of very low population size (so that values observed would be less than this). For an illustrative reference case (see Table 2), pregnancy rate is assumed to be the only (linearly) density dependent demographic parameter, decreasing to $\rho_{\min }=0.1$ when the population is at carrying capacity. Other biological parameters for this reference case are taken to be fixed (density independent); age at first parturition $a^{\text {mat }}=5$ years and an annual natural mortality rate $M=0.03 \mathrm{yr}^{-1}$.

Sensitivity to changes in these assumptions is then examined, first for single factors, and then for these factors in combination. Initially four factors are considered:

(A) Density dependence (linear) in the age at first parturition, first with $a_{\text {max }}^{\text {mat }}=10$;

(B) Lower values for natural mortality;

(C) Nonlinear density dependence for $\rho$ and $a^{\text {mat }}$ reflected by the parameter $\mu$ (see Appendix equations A.14 and A.15); note that $\mu=1$ reflects linear dependence, and $\mu$ $>1$ means that density dependent effects do not come into play as rapidly when the population grows from a low level, so that high growth rates can be sustained through to larger population sizes;

(D) Density dependence (linear) in natural mortality (Appendix equation A.16).

Not all combinations of parameter values are feasible. The condition of a steady population at carrying capacity $K$ leads to restrictions on the value of natural mortality for the first year $\left(M_{0}\right)$. Clearly $M_{0}$ cannot be negative. The computations reported below insist further that always $M_{0} \geq M_{1}$ where $M_{1}$ is the natural mortality of age 1 humpback whales (which is identical to that of all older humpbacks for scenarios without
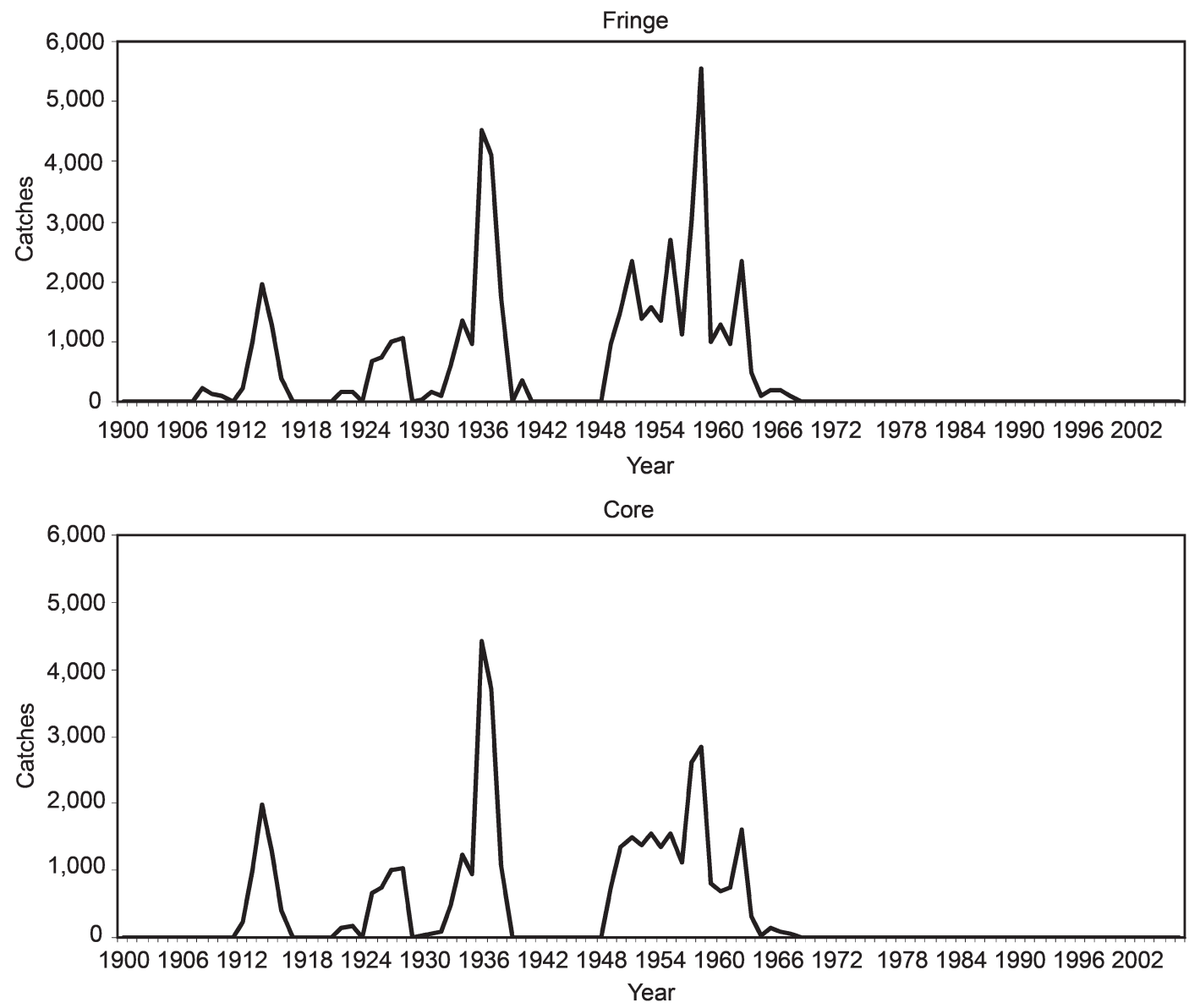

Fig. 1. Catch series for Breeding Stock D humpback whales for the Fringe (above) and Core (below) catch allocation hypotheses (IWC, 2011). 
Table 2

Description of model variants and how they are referenced in the paper. The model indicated in brackets ( ) refers to the model of which the model under consideration is a variant; under description, what has been changed in the model under consideration is shown underlined. See the Appendix for detailed definitions of the symbols. The value calculated for natural mortality for the first year of life $\left(M_{0}\right)$ (see equations A.11 and A.12) is also given, together with an asterisk (*) if it is limited by the constraint $M_{0}>M_{1}$.

\begin{tabular}{|c|c|c|c|}
\hline Model & Name & Description & $M_{0}$ \\
\hline 1 & Reference case & $a_{\max }^{\operatorname{mat}}=5 ; \rho_{\min }=0.1 ; \mu=1 ; M_{m}^{\min }=M_{m}^{\max }=0.03$ & 0.348 \\
\hline $2 \mathrm{a}(\mathrm{ref})$ & $a_{\max }^{\text {mat }}=10$ & $a_{\max }^{\operatorname{mat}}=\underline{10} ; \rho_{\min }=0.1 ; \mu=1 ; M_{m}^{\min }=M_{m}^{\max }=0.03$ & 0.188 \\
\hline $2 b($ ref) & $M_{m}=0.02$ & $a_{\max }^{\max t}=5 ; \rho_{\min }=0.1 ; \mu=1 ; M_{m}^{\min }=M_{m}^{\max }=\underline{0.02}$ & 0.688 \\
\hline $2 \mathrm{c}(\mathrm{ref})$ & $\mu=3$ & $a_{\max }^{\operatorname{mat}}=5 ; \rho_{\min }=0.1 ; \mu=\underline{3} ; M_{m}^{\min }=M_{m}^{\max }=0.03$ & 0.348 \\
\hline $2 \mathrm{~d}(\mathrm{ref})$ & $M_{m}: 0.03 \rightarrow 0.02$ & $a_{\max }^{\operatorname{mat}}=5 ; \rho_{\min }=0.1 ; \mu=1 ; M_{m}^{\min }=\underline{0.02} ; M_{m}^{\max }=\underline{0.03}$ (i.e. $M_{m}$ density dependent) & 0.348 \\
\hline $3 a(r e f)$ & All changes & $a_{\max }^{\operatorname{mat}}=\underline{10} ; \rho_{\min }=0.1 ; \mu=\underline{3} ; M_{m}^{\min }=\underline{0.02} ; M_{m}^{\max }=\underline{0.03}$ & 0.188 \\
\hline $3 b(3 a)$ & $3 \mathrm{a}+\max M_{m}$ decr. & $a_{\max }^{\max }=10 ; \rho_{\min }=0.1 ; \mu=3 ; M_{m}^{m i n}=0.02 ; M_{m}^{\max }=\underline{0.034}$ (i.e. maximal $M_{m}$ density dependence possible) & 0.050 \\
\hline $3 c(3 b)$ & $3 b+$ extreme values & $a_{\max }^{\max }=\underline{12} ; \rho_{\min }=0.1 ; \mu=\underline{5} ; M_{m}^{m i n}=\underline{0.01} ; M_{m}^{\max }=\underline{\underline{0.032}}$ & 0.051 \\
\hline 4ai (ref) & Ref $-M$ incr. with age & $a_{\max }^{\operatorname{mat}}=5 ; \rho_{\min }=0.1 ; \mu=1 ; M_{m}^{\min }=M_{m}^{\max }=0.03 ; M_{h}-M_{m}=\underline{0.05} ; a_{2}=\underline{30} ; a_{3}=\underline{40}$ & 0.118 \\
\hline 4aii (ref) & Ref $-\max M$ incr. with age & $a_{\max }^{\operatorname{mat}}=5 ; \rho_{\min }=0.1 ; \mu=1 ; M_{m}^{\min }=M_{m}^{\max }=0.03 ; M_{h}-M_{m}=\underline{0.05} ; a_{2}=\underline{23} ; a_{3}=\underline{33}$ & 0.038 \\
\hline $4 \mathrm{bi}(3 \mathrm{a})$ & $3 \mathrm{a}-M$ incr. with age & $a_{\max }^{a_{\max }}=10 ; \rho_{\min }=0.1 ; \mu=3 ; M_{m}^{\min }=0.02 ; M_{m}^{\max }=0.03 ; M_{h}-M_{m}=\underline{0.02} ; a_{2}=\underline{30} ; a_{3}=\underline{40}$ & 0.039 \\
\hline 4bii (3a) & $3 \mathrm{a}-\max M$ incr. with age & $a_{\max }^{\operatorname{mat}}=10 ; \rho_{\min }=0.1 ; \mu=3 ; M_{m}^{\min }=0.02 ; M_{m}^{\max }=0.03 ; M_{h}-M_{m}=0.02 ; a_{2}=\underline{29} ; a_{3}=\underline{39}$ & 0.032 \\
\hline $4 c(3 b)$ & $3 \mathrm{~b}-M$ incr. with age & $a_{\max }^{\operatorname{mat}}=10 ; \rho_{\min }=0.1 ; \mu=3 ; M_{m}^{m i n}=0.02 ; M_{m}^{\max }=\underline{0.0302} ; M_{h}-M_{m}=\underline{0.02} ; a_{2}=\underline{30} ; a_{3}=\underline{40}$ & 0.033 \\
\hline $4 d(3 c)$ & $3 \mathrm{c}-M$ incr. with age & $a_{\max }^{\operatorname{mat}}=12 ; \rho_{\min }=0.1 ; \mu=5 ; M_{m}^{\min }=0.01 ; M_{m}^{\max }=\underline{0.028} ; M_{h}-M_{m}=\underline{0.02} ; a_{2}=\underline{30} ; a_{3}=\underline{40}$ & $0.028^{*}$ \\
\hline $5 \mathrm{a}(3 \mathrm{c})$ & $3 \mathrm{c}+$ preg $=0.6$ & $a_{\max }^{\max }=12 ; \rho_{\min }=0.1 ; \mu=5 ; M_{m}^{\min }=0.01 ; M_{m}^{\max }=0.032 ; \gamma_{\max }=\underline{0.6}$ & 0.051 \\
\hline 6ai & Ref $-17,959$ target & $a_{\max }^{\operatorname{mat}}=5 ; \rho_{\min }=0.1 ; \mu=1 ; M_{m}^{\min }=M_{m}^{\max }=0.03 ; N_{1997}^{T}=\underline{17,959}$ & 0.348 \\
\hline 6aii & Ref $-31,750$ target & $a_{\max }^{\operatorname{mat}}=5 ; \rho_{\min }=0.1 ; \mu=1 ; M_{m}^{\min }=M_{m}^{\max }=0.03 ; N_{2003}^{T}=\underline{31,750}$ & 0.348 \\
\hline $6 \mathrm{bi}$ & $3 a-17,959$ target & $a_{\max }^{\operatorname{mat}}=10 ; \rho_{\min }=0.1 ; \mu=3 ; M_{m}^{\min }=0.02 ; M_{m}^{\max }=0.03 ; N_{1997}^{T}=\underline{17.959}$ & 0.188 \\
\hline 6 bii & $3 a-31,750$ target & $a_{\max }^{\operatorname{mat}}=10 ; \rho_{\min }=0.1 ; \mu=3 ; M_{m}^{m i n}=0.02 ; M_{m}^{\max }=0.03 ; N_{2003}^{T}=\underline{31,750}$ & 0.188 \\
\hline $7 \mathrm{bi}$ & $6 \mathrm{bi}+K^{*}=1.5 K$ & $a_{\max }^{\operatorname{mat}}=10 ; \rho_{\min }=0.1 ; \mu=3 ; M_{m}^{\min }=0.02 ; M_{m}^{\max }=0.03 ; N_{1997}^{T}=17,959 ; K^{*}=\underline{1.5 K}$ & 0.188 \\
\hline 7 bii & $6 \mathrm{bii}+K^{*}=2 K$ & $a_{\max }^{\operatorname{mat}}=10 ; \rho_{\min }=0.1 ; \mu=3 ; M_{m}^{\min }=0.02 ; M_{m}^{\max }=0.03 ; N_{2003}^{T}=31,750 ; K^{*}=\underline{2 K}$ & 0.188 \\
\hline
\end{tabular}

age dependence in $M$ ) (see Appendix equations A.11 and A.12 and associated text).

The possibility that $M$ increases at larger ages is implemented through equation A.13. Potentially this could introduce temporary high growth rates if the population for a time includes an over-representation of younger animals past the age at first parturition as it recovers from heavy depletion.

Other factors investigated are alternative target population levels and a change in carrying capacity. The alternative levels are: 31,750 for 2003 from JARPA surveys (Matsuoka et al., 2011); and 17,959 for 1997 from the IDCR surveys (Branch, 2011).

An increase in $K$ over the period 1930 to 1960 is considered as a manifestation of possible competitive release (as regards utilisation of krill) arising from the major reduction of blue and fin whales over that period as a result of harvesting.

\section{RESULTS AND DISCUSSION}

Table 2 lists the specifications of the reference case and other models implemented for Breeding Stock D, together with shortened names for each for ease of reference. It also lists the value of $M_{0}$ for each model, indicating cases where the $M_{0} \geq M_{1}$ constraint has come into play.

Results are shown in Table 3 as annual rates of population growth for each model for three periods: the first five (196872) and first ten (1968-77) years after catching ceased (these are the periods where growth rates might be expected to be highest as the population is at its lowest level), and the 19821994 period over which breeding area surveys lead to an annual rate of increase estimate of 0.10 .

Immediately evident from Table 3 is that there is very little difference between results for the Core and the Fringe catch allocation hypotheses. Hence the discussion that follows focuses on results for the Fringe case only.
For virtually all the models considered, the rate of population increase over the first ten years since catches ceased is greater than that over the first five years (the exceptions are for the higher JARPA and IDCR survey based target population sizes). The primary reason for this is likely the time lag until calves reach the age at which they can themselves reproduce and add to the population. In most cases increase rates over 1982-94 are less than those over the first ten years since catches ceased. In cases where this is not so (i.e. transient effects are sufficient to compensate for the opposite impact from density dependence), the differences are marginal. Further discussion focuses on the 1982-94 rates only, as this is the period for which an estimate is available from the survey series.

The reference case reflects an annual population increase rate of 0.070 over 1982-94. Density dependence in the age at first parturition ( $a^{\text {mat }}$ ranging from 5 to 10 years) and in the natural mortality $M$ (ranging from 0.02 to $0.03 \mathrm{yr}^{-1}$ ) each separately increase this by about 0.01 , as does nonlinearity in the density dependence ( $\mu$ changed from 1 , corresponding to linear dependence, to 3$)$. The fact that lowering $M$ from 0.03 to 0.02 in the absence of density dependence in $M$ leads to a drop in the increase rate may seem surprising; it arises from the fact that balancing births and deaths at carrying capacity given a lower $M$ value, requires an increase in $M_{0}$, which in turn reduces the rate at which the population can grow when reduced in abundance.

All of these changes together (model 3a) see the annual increase rate raised to 0.10 (which coincidentally happens to be the point estimate from the observations). If the extent to which $M$ can change with density is maximised subject to the constraint that $M_{0}>M$ (model $\left.3 \mathrm{~b}\right)$, the rate increases to 0.11. Finally if more extreme (but not impossible) ranges/values are used ( $a^{\text {mat }}: 5$ to $12 ; \mu=5 ; M: 0.01$ to 0.032 - model $3 \mathrm{c}$ ), a rate of 0.12 can be realised. Fig. 2 illustrates the population trajectories for a number of these cases. 
Table 3

Annual rates of increase for the model variants considered. The periods considered are the first five (1968-72) and ten (1968-77) years after catches dropped to zero, and then the 1982-1994 period over which a relative abundance index is available for the breeding grounds which indicates an annual increase rate of 0.101 (95\% CI: [0.028; 0.175]). 'Maximum' gives the maximum steady growth rate possible given the biological parameter values for the model in question. Models marked with an asterisk were fitted with age-at-first capture $\left(a_{r}\right)$ of 2 rather than 4 years for reasons explained in the text. A double asterisk indicates that the change in the value of this parameter applies only to the Fringe catch allocations.

\begin{tabular}{|c|c|c|c|c|c|c|c|c|}
\hline \multirow[b]{2}{*}{ Model } & \multirow[b]{2}{*}{ Name } & \multicolumn{3}{|c|}{ Core } & \multicolumn{4}{|c|}{ Fringe } \\
\hline & & $68-72$ & $68-77$ & $82-94$ & $68-72$ & $68-77$ & $82-94$ & Maximum \\
\hline 1 & Reference case & 0.076 & 0.076 & 0.066 & 0.075 & 0.077 & 0.070 & 0.081 \\
\hline $2 \mathrm{a}(\mathrm{ref})$ & $a_{\max }^{\operatorname{mat}}=10$ & 0.087 & 0.085 & 0.069 & 0.086 & 0.087 & 0.075 & 0.093 \\
\hline $2 b$ (ref) & $M_{m}^{\max }=0.02$ & 0.062 & 0.062 & 0.055 & 0.063 & 0.063 & 0.058 & 0.067 \\
\hline $2 \mathrm{c}$ (ref) & $\mu \stackrel{m}{=} 3$ & 0.079 & 0.080 & 0.079 & 0.078 & 0.080 & 0.080 & 0.081 \\
\hline 2d (ref) & $M_{m}: 0.03 \rightarrow 0.02$ & 0.085 & 0.085 & 0.073 & 0.084 & 0.086 & 0.078 & 0.090 \\
\hline $3 b(3 a) *$ & $3 \mathrm{a}+\max M$ decr. & 0.110 & 0.112 & 0.108 & 0.105 & 0.111 & 0.110 & 0.113 \\
\hline $3 c(3 b)^{*}$ & $3 \mathrm{~b}+$ extreme values & 0.118 & 0.120 & 0.118 & 0.111 & 0.119 & 0.119 & 0.122 \\
\hline 4ai (ref) & Ref $-M$ incr. with age & 0.093 & 0.094 & 0.082 & 0.091 & 0.094 & 0.087 & 0.098 \\
\hline 4aii (ref)** & Ref $-\max M$ incr. with age & 0.099 & 0.100 & 0.088 & 0.098 & 0.101 & 0.092 & 0.104 \\
\hline $4 b i(3 a)^{*}$ & $3 \mathrm{a}-M$ incr. with age & 0.111 & 0.113 & 0.110 & 0.105 & 0.111 & 0.112 & 0.114 \\
\hline 4 bii $(3 a) *$ & $3 \mathrm{a}-\max M$ incr. with age & 0.112 & 0.113 & 0.110 & 0.106 & 0.112 & 0.112 & 0.114 \\
\hline $4 c(3 b)^{*}$ & $3 \mathrm{~b}-M$ incr. with age & 0.112 & 0.113 & 0.110 & 0.105 & 0.112 & 0.112 & 0.114 \\
\hline $4 d(3 c)^{*}$ & $3 c-M$ incr. with age & 0.120 & 0.122 & 0.120 & 0.112 & 0.120 & 0.121 & 0.124 \\
\hline $6 a i$ & Ref $-17,959$ target & & & & 0.072 & 0.071 & 0.054 & 0.081 \\
\hline 6aii & Ref $-31,750$ target & & & & 0.056 & 0.052 & 0.024 & 0.081 \\
\hline $6 \mathrm{bi}$ & $3 a-17,959$ target & & & & 0.099 & 0.100 & 0.085 & 0.102 \\
\hline 6bii & $3 a-31,750$ target & & & & 0.037 & 0.025 & 0.001 & 0.102 \\
\hline $7 \mathrm{bi}$ & $6 \mathrm{bi}+K^{*}=1.5 K$ & & & & 0.099 & 0.101 & 0.095 & 0.102 \\
\hline 7 bii & $6 \mathrm{bii}+K^{*}=2 K$ & & & & 0.100 & 0.101 & 0.090 & 0.102 \\
\hline
\end{tabular}

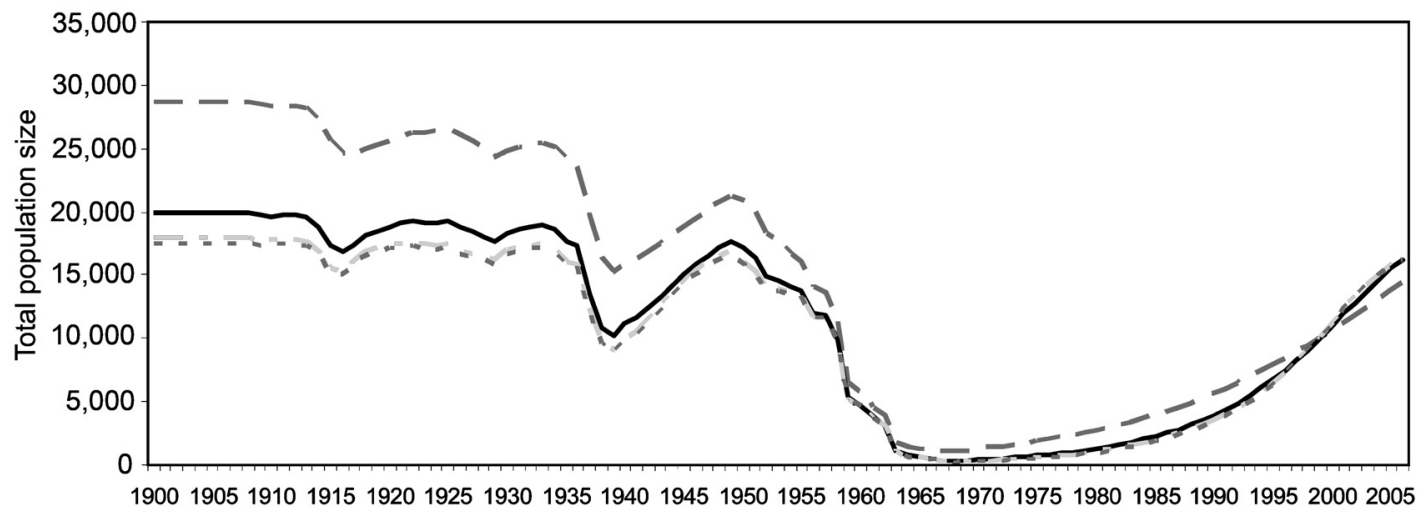

Year

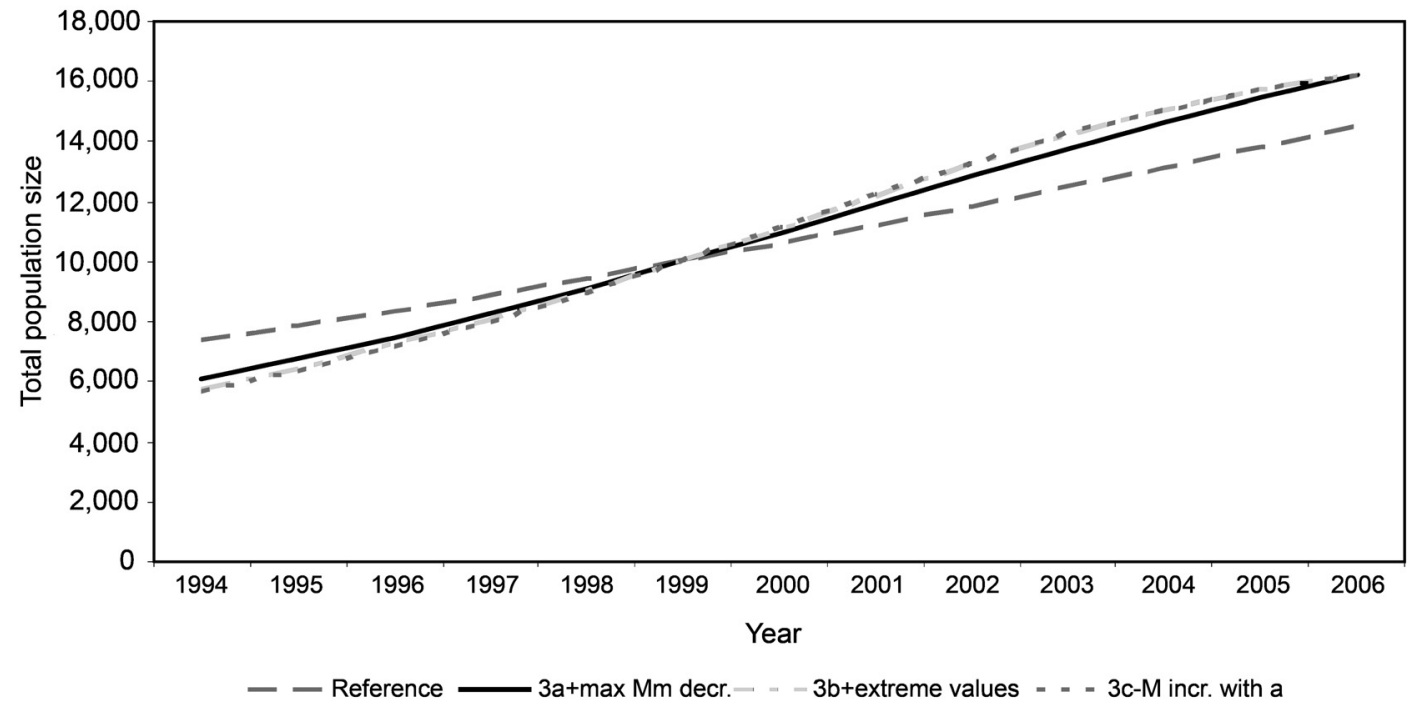

Fig. 2. Comparison of population trajectories for stock D humpback whales for the reference case model, and variants of this model in which all the parameters are changed. Trajectories are shown for the whole period since exploitation commenced (top) and for the 1994-2006 period only (bottom). 

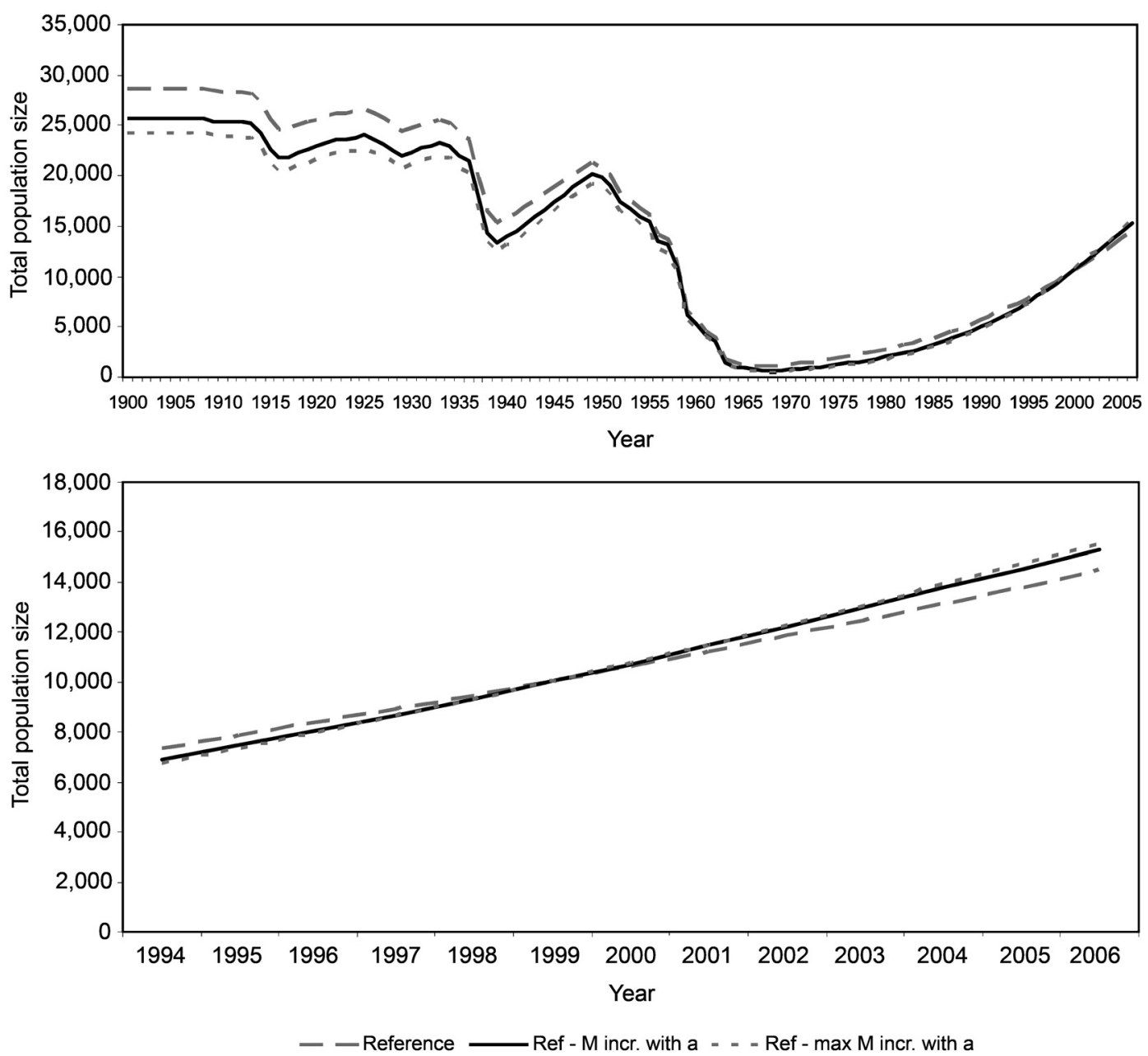

Fig. 3. Comparison of population trajectories for stock D humpback whales for the reference case model, and variants of this model which allow for various degrees of larger natural mortality at older ages. Trajectories are shown for the whole period since exploitation commenced (top) and for the 1994-2006 period only (bottom)

If higher natural mortality at larger ages is allowed (models 4ai and 4aii), the increase rate for the reference case becomes larger by about 0.02 (see Fig. 3). However, such higher mortality in combination with more extreme ranges for the other parameters (model 4d) can achieve only little extra increase in the growth rate. These increases are limited essentially because the $M_{0}>M_{1}$ constraint comes into play (see Table 3 ), and precludes more extreme choices for the $M_{m}^{\max }, a_{2}$ and $a_{3}$ parameters.

Constraining the maximum pregnancy rate to 0.5 has an influential effect. Were annual compared to biennial calvings sufficiently frequent to increase the average maximum pregnancy rate from 0.5 to 0.6 (model $5 \mathrm{a})$, the population annual increase rate could approach $14 \%$.

In most instances with higher target levels for recent abundance (from JARPA or IDCR surveys - models 6), the population shows a relatively low rate of increase over 1982 to 1994 , essentially because it is estimated to be close to carrying capacity by the start of that period. However the fact that the populations are never reduced to a very low level under some such scenarios (see Fig. 4) raises questions about their plausibility. This inconsistency can, however, be resolved if an increase in carrying capacity is postulated (model 7). An increase of $K$ of $50 \%$ or $100 \%$ for target levels of, respectively, 17,959 in 1997 or 31,750 in 2003 sees annual increase rates back to near 0.10 (Fig. 4).

For all models considered the maximum steady growth rate possible given the biological parameters for the model in question is greater than the predicted rate of increase over 1982 to 1994 . Thus these analyses provide no indication that the point estimate of the rate of increase observed can be reconciled with less extreme values of demographic parameters than led to the original $r=0.126$ upper bound as a consequence of transient effects.

\section{CONCLUSION}

Unless the possibilities (in the limit of low population size) of an age at first parturition less than five years, or an average pregnancy rate above 0.5 can be entertained, it seems that 0.12 is about the maximum demographically feasible annual increase rate for a closed population of Breeding Stock D humpback whales over the 1982-94 period. Transient effects do not appear able to reconcile the observed rate of increase with less extreme values of demographic parameters than led to the previously imposed upper bound of 0.126 on the maximum possible annual growth rate ${ }^{1}$.

\footnotetext{
${ }^{1}$ Subsequent to the original presentation of this paper, the IWC Scientific Committee (IWC, 2007) agreed that it was reasonable to revise down the upper bound on the maximum annual increase rate from the earlier 0.126 to 0.106 , based on extreme values reported for Northern Hemisphere humpback whale populations for pregnancy rate, age at first parturition and natural mortality, though noting that the more extreme values still in the limit of very low population size could not be excluded. It is, however, unlikely that use of those Northern Hemisphere extreme values rather than those considered in this paper would change the conclusion that transient effects do not have an appreciable impact on the population growth rates observed.
} 


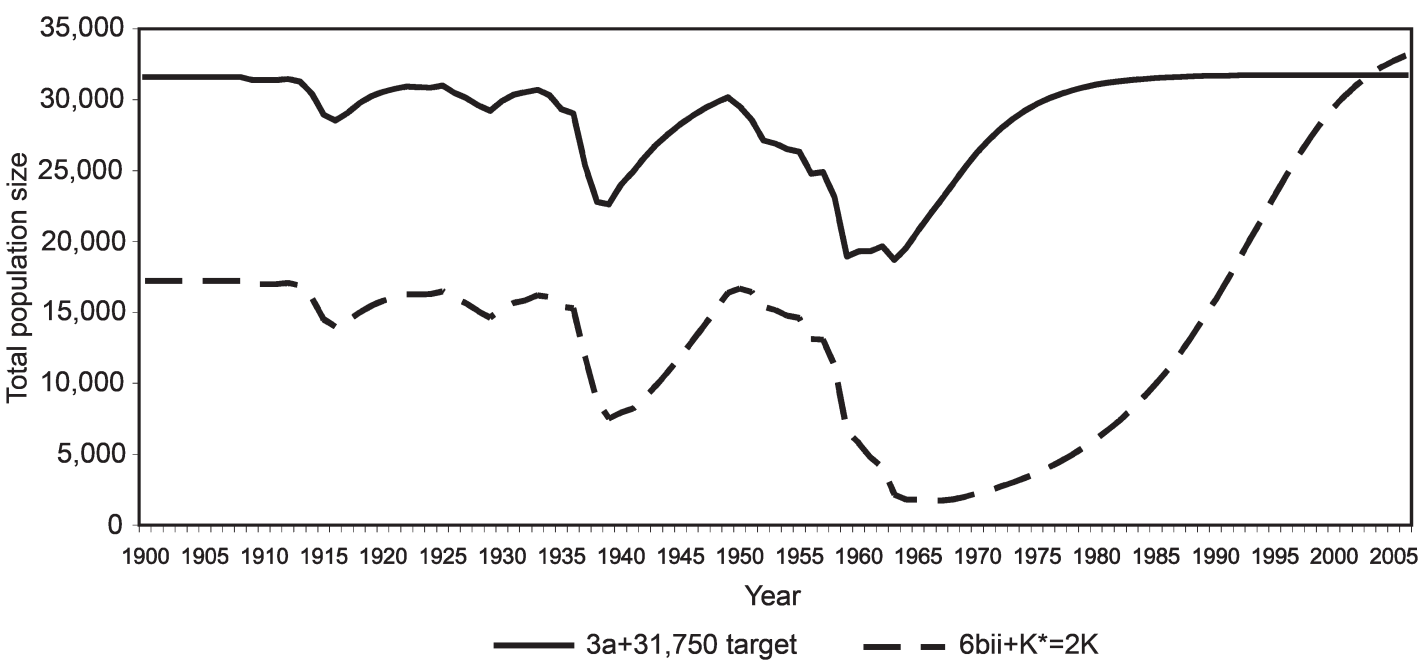

Fig. 4. Comparison of population trajectories for stock D humpback whales for the ' $3 a$ ' variant of the reference case model with a target population of 31,750 in 2003 , and for this model with the inclusion of a $100 \%$ increase in carrying capacity from 1930 to 1960

\section{ACKNOWLEDGEMENTS}

The authors thank Andre Punt and an anonymous reviewer for their comments on an earlier version of this paper. Financial support for this work by the South African National Research Foundation is gratefully acknowledged.

\section{REFERENCES}

Branch, T.A. 2011. Humpback whale abundance south of $60^{\circ} \mathrm{S}$ from three complete circumpolar sets of surveys. J. Cetacean Res. Manage. (special issue 3): 53-69.

Brandāo, A., Butterworth, D.S. and Brown, M.R. 1999. Maximum possible humpback whale increase rates as a function of biological parameter values. J. Cetacean Res. Manage. (Suppl.) 2: 192-93.

Chittleborough, R.G. 1965. Dynamics of two populations of the humpback whale, Megaptera novaeangliae (Borowski). Aust. J. Mar. Freshwater Res. 16(1): 33-128

Clapham, P., Robbins, J., Brown, M., Wade, P. and Findlay, K. 2001. Report of the Scientific Committee. Annex G. Report of the Sub-Committee on the Comprehensive Assessment of Whale Stocks - In-depth Assessments. Appendix 5. A note on plausible rates of population growth in humpback whales. J. Cetacean Res. Manage. (Suppl.) 3: 196-97.

International Whaling Commission. 1996. Report of the Scientific Committee, Annex E. Report of the sub-committee on Southern Hemisphere baleen whales. Rep. int. Whal. Commn 46:117-38.

International Whaling Commission. 2007. Report of the Scientific Committee. Annex H. Report of the Sub-Committee on Other Southern Hemisphere Whale Stocks. J. Cetacean Res. Manage. (Suppl.) 9:188209.

International Whaling Commission. 2011. Report of the Workshop on the Comprehensive Assessment of Southern Hemisphere humpback whales, 4-7 April 2006, Hobart, Tasmania. J. Cetacean Res. Manage. (special issue 3): 1-50.

Matsuoka, K., Hakamada, T., Kiwada, H., Murase, H. and Nishiwaki, S. 2011. Abundance estimates and trends for humpback whales (Megaptera novaeangliae) in Antarctic Areas IV and V based on JARPA sightings data. J. Cetacean Res. Manage. (special issue 3): 75-94.

Paxton, C.G.M., Hedley, S.L. and Bannister, J.L. 2011. Group IV humpback whales: their status from aerial and land-based surveys off Western Australia, 2005. J. Cetacean Res. Manage. (special issue 3): 223-234.

\section{Appendix}

\section{DETAILS OF THE AGE-STRUCTURED POPULATION MODEL}

\section{Population dynamics}

where:

$$
\begin{array}{ll}
N_{\mathrm{y}+1,1}=0.5 \rho\left(N_{y}^{T}\right) e^{-M_{0}} N_{y}^{m} & y \geq 0 \\
N_{\mathrm{y}+1, a+1}=\left(N_{y, a}-C_{y, a}\right) e^{-M_{a}} & 1 \leq a \leq 99
\end{array}
$$

$N_{v, a}$ is the number of humpback whales of age $a$ in year $y$,

$C_{y, a}$ is the number of humpback whales of age $a$ caught in year $y$,

$M_{a}$ is the natural mortality rate at age $a$,

$N_{y}^{T}$ is total population in year $y$ (defined to be one year old and older humpback whales) given by:

$$
N_{y}^{T}=\sum_{a=1}^{100} N_{y, a},
$$


$N_{y}^{m}$ is the mature population of humpback whales in year $y$ given by:

$$
N_{y}^{m}=\sum_{a=1}^{100} N_{y, a} \gamma_{a},
$$

where:

$\gamma_{\mathrm{a}}$ is the fraction of humpback whales of age $a$ that are mature, given by:

$$
\gamma_{a}=\left\{\begin{array}{cc}
0 & a<\operatorname{int}\left(a^{\text {mat }}\left(N_{y}^{T}\right)\right) \\
1-\left(a^{\text {mat }}\left(N_{y}^{T}\right)-a\right) & a=\operatorname{int}\left(a^{\text {mat }}\left(N_{y}^{T}\right)\right), \\
1 & a>\operatorname{int}\left(a^{\text {mat }}\left(N_{y}^{T}\right)\right)
\end{array}\right.
$$

where:

$a^{\text {mat }}\left(N_{y}^{T}\right)$ is the age at first parturition, as a function of the total population size, given by:

$$
a^{\text {mat }}\left(N_{y}^{T}\right)=\left(a_{\max }^{\text {mat }}-a_{\min }^{\text {mat }}\right) N_{y}^{T} / K_{y}+a_{\min }^{\text {mat }},
$$

where:

$a_{\max }^{\text {mat }}$ is the maximum age at first parturition,

$a_{\min }^{\text {mat }}$ is the minimum age at first parturition, and

$K_{y}$ is the carrying capacity, which can change linearly over the years 1930 to 1960 from $K$ to $K^{*}$ :

$$
K_{y}=\left\{\begin{array}{cc}
K & 1900 \leq y<1930 \\
K+\left(K^{*}-K\right) \frac{(y-1930)}{30} & 1930 \leq y \leq 1960 \\
K^{*} & 1960<y \leq 2006
\end{array},\right.
$$

$\rho\left(N_{y}^{T}\right)$ is the pregnancy rate, which depends on the total population size, and given by:

$$
\rho\left(N_{y}^{T}\right)=\rho_{\max }-\left(\rho_{\max }-\rho_{\min }\right)^{N_{y}^{T}} / K_{y},
$$

where:

$\rho_{\min }$ is the minimum pregnancy rate, and

$\rho_{\max }$ is the maximum pregnancy rate.

Note that it is assumed that all humpback whales reaching the age of 100 then immediately die. Given that the only instances of evidence of whales living beyond 100 is for bowheads, setting 100 as a maximum age seems reasonable.

The number of whales of age $a$ caught in year $y$ is given by:

$$
C_{y, a}=\left\{\begin{array}{cc}
0 & a<a_{r} \\
C_{y} \frac{N_{y, a}}{\sum_{a^{\prime}=a_{r}}^{99} N_{y, a^{\prime}}} & a \geq a_{r}
\end{array}\right.
$$

where:

$a_{r}$ is the age at first capture, and

$C_{y}$ is the catch in year $y$.

i.e. uniform selectivity for ages $a_{r}$ and above is assumed.

The initial numbers at each age $a$ are taken to follow an unexploited equilibrium distribution evaluated as follows:

where:

$$
N_{0, \mathrm{a}}=\lambda N_{a}^{*} \text { for } a=1, \ldots, 100,
$$

$$
\lambda=\frac{K}{\sum_{a=1}^{100} N_{a}^{*}},
$$


$K$ is the (initial) carrying capacity.

$$
N_{1}^{*}=1, N_{2}^{*}=N_{1}^{*} e^{-M_{1}}, N_{3}^{*}=N_{2}^{*} e^{-M_{2}}, \text { etc., and }
$$

\section{Natural mortality}

The natural mortality for the first year of life $\left(M_{0}\right)$ is calculated by ensuring that the number of calves that reach age one each year balances the number of deaths (of humpback whales of age one and above) per year when the population is unexploited (i.e. when $N_{0}^{T}=K$ ). In this instance the number of such calves is proportional to (taking $a_{\max }^{\text {mat }}$ here to be integral):

$$
0.5 \rho_{\min } e^{-M_{0}} \sum_{a=a_{\max }^{\operatorname{mat}}}^{100} N_{a}^{*}
$$

and the number of deaths is in the same proportion to:

$$
\sum_{a=1}^{99} N_{a}^{*}\left(1-e^{-M_{a}}\right)+N_{100}^{*}
$$

so that $M_{0}$ can be computed by equating A.11 to A.12.

The natural mortality for the first year of life $\left(M_{0}\right)$ is constrained to be greater than the natural mortality of one year olds $\left(M_{l}\right)$. To allow for the possibility of increased natural mortality at older ages, the natural mortality at age is modelled to change smoothly by:

$$
M_{a}=\left\{\begin{array}{cc}
M_{m} & 1 \leq a<a_{2} \\
M_{m}+\left(M_{h}-M_{m}\right) \frac{\left(a-a_{2}\right)}{\left(a_{3}-a_{2}\right)} & a_{2} \leq a \leq a_{3} \\
M_{h} & a_{3}<a \leq 100
\end{array},\right.
$$

where:

$M_{m}$ is the lowest value for natural mortality, and

$M_{h}$ is the highest value for natural mortality with $M_{m}$ increasing linearly to $M_{h}$ over the age range $a_{2}$ to $a_{3}$.

When the value for $M_{h}$ is set the same as that for $M_{m}$, natural mortality is independent of age.

\section{Alternative forms of density dependence}

Alternative formulations are considered to allow for density dependence in different forms in the calculation of age at first parturition $\left(a^{m a t}\left(N_{y}^{T}\right)\right)$, the pregnancy rate $\left(\rho\left(N_{y}^{T}\right)\right)$ and the natural mortality $M_{a}$. Equation (A.4) is changed to:

$$
a^{\text {mat }}\left(N_{y}^{T}\right)=\left(a_{\max }^{\text {mat }}-a_{\min }^{\text {mat }}\right)\left(N_{y}^{T} / K_{y}\right)^{\mu}+a_{\min }^{\text {mat }},
$$

equation (A.6) is changed to:

$$
\rho\left(N_{y}^{T}\right)=\rho_{\text {max }}-\left(\rho_{\max }-\rho_{\min }\right)\left(N_{y}^{T} / K_{y}\right)^{\mu} \text {, and }
$$

the lowest value for natural mortality $\left(M_{m}\right)$ in equation (A.13) is changed to:

$$
M_{m}=M_{m}^{\min }+\left(M_{m}^{\max }-M_{m}^{\min }\right)^{N_{y}^{T}} / K_{y} .
$$

Fig. A.1 illustrates how different choices for $\mu$ affect the dependence of pregnancy rate on population abundance.

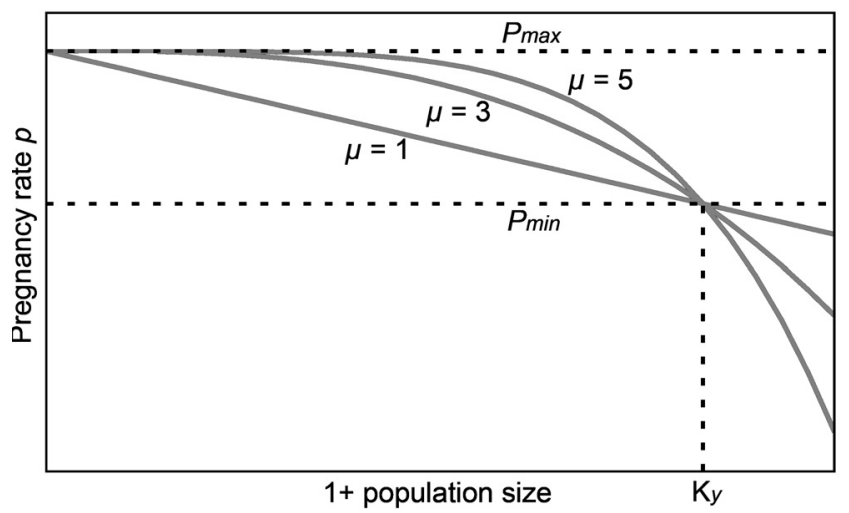

Fig. A.1. The variation of pregnancy rate with total population size in relation to the value of the $\mu$ parameter (see equation A.15). 